\title{
Effects of Carnosine and Beta-Alanine Ingestion on Anaerobic Sprint Performance and Peripheral Blood Mononuclear Cell Interleukin-6 and -10 Gene Expression
}

\author{
Pietro Luigi Invernizzi ${ }^{1}$, Bruno Venerando ${ }^{2}$, Francesco Di Pierro ${ }^{3}$, \\ Sandro Saronni ${ }^{4}$, Nadia Papini ${ }^{2}$ \\ ${ }^{1}$ Department of Biomedical Science for Health, University of Milan, Milan, Italy \\ ${ }^{2}$ Department of Medical Biotechnology and Translational Medicine, University of Milan, Milan, Italy \\ ${ }^{3}$ Velleja Research, Pontenure, Piacenza, Italy \\ ${ }^{4}$ School of Sports Science, University of Milan, Milan, Italy \\ Email: pietro.invernizzi1@unimi.it
}

Received September $25^{\text {th }}, 2013$; revised October $25^{\text {th }}, 2013$; accepted November $2^{\text {nd }}, 2013$

Copyright (C 2013 Pietro Luigi Invernizzi et al. This is an open access article distributed under the Creative Commons Attribution License, which permits unrestricted use, distribution, and reproduction in any medium, provided the original work is properly cited.

\begin{abstract}
Chronic administration of $\beta$-alanine has been shown to increase muscle carnosine content and improve anaerobic performance. It is not clear whether acute ingestion of carnosine and beta alanine may have the same effects. With a view to investigating acute effects of carnosine and $\beta$-alanine ingestion on anaerobic intermittent running performance and on the responses of Interleukin- 6 and -10 to exercise, twelve healthy, young, active participants (age: $21 \pm 4$ years) underwent the running-based anaerobic test (RAST) twice (with $30 \mathrm{~min}$ recovery in between) on two separate occasions (randomized, crossover design). The test consisted of $6 \times 35-\mathrm{m}$ sprints interspersed with $10 \mathrm{~s}$ rests after acute ingestion ( 4 hours before the test) of either $2 \mathrm{~g} \mathrm{~L}$-carnosine $+2 \mathrm{~g} \beta$-alanine or placebo. The overall performance decreased (RAST1 vs RAST2, carnosine $+\beta$-alanine: $32.8 \pm 1.3 \mathrm{~s}, 33.4 \pm 1.2 \mathrm{~s}$; Placebo: $32.9 \pm 1.0 \mathrm{~s}, 33.6 \pm 1.2 \mathrm{~s}$ ), pain after RASTs increased (RAST1 vs RAST2, carnosine $+\beta$-alanine: $3.0 \pm 2.1$ a.u., $4.2 \pm 1.9$ a.u.; Placebo: $3.0 \pm$ 1.8 a.u., $3.4 \pm 1.2$ a.u.) almost in the same way in both groups, and RPE did not show any difference. IL6 and IL10 gene expression increased and decreased respectively in response to exercise in the same fashion in both conditions. During RAST 2 we found a potentially increased performance in the carnosine + $\beta$-alanine group (main effect of condition, $p<0.05$ ). In conclusion these findings suggest that acute administration of carnosine $+\beta$-alanine does not influence the cytokine response to exercise but might have a very small enhancing effect on anaerobic sprint performance.
\end{abstract}

Keywords: Running; Supplementation; Cytokines

\section{Introduction}

Dipeptide carnosine ( $\beta$-alanyl-L-histidine) is found in various tissues such as brain and heart, but especially in skeletal muscle fibres (Harris et al., 2012) of both types I and II, where carnosine concentration is the highest (Baguet et al., 2010). Although carnosine is synthesized endogenously, its tissue concentration is influenced by the diet (Baguet et al., 2010). Indeed, exogenous supplementation of $\beta$-alanine (precursor and limiting factor in the synthesis of carnosine) has been shown to increase muscle carnosine concentration in vitro (Margolis et al., 1985) and in animal models (Hill et al., 2007). Beta-alanine is usually administered orally and for a period of 1 - 4 weeks (Jagim et al., 2013), since its chronic consumption seems to be necessary to ensure carnosine increase inside muscle fibres (Derave et al., 2007).

Carnosine levels seem to be correlated to exercise performance: Suzuki et al (Suzuki et al., 2002) have observed a positive correlation between carnosine content in the vastus lateralis and the power generated at the end of a 30 -second cycle ergometer test (Wingate test) in untrained individuals.
Hill et al. (Hill et al., 2007) demonstrated that $\beta$-alanine administration in active males produced a significant increase in carnosine levels in skeletal muscles, which was related to an improvement of exercise performance. In particular, $\beta$-alanine was administered to untrained males for 10 weeks, producing not only a remarkable increase in carnosine content in the vastus lateralis, but also a significant prolongation of the time to exhaustion during a cycling test performed at $110 \%$ of energy production and maximal heart rate, where the resistance time is estimated to be about 2.5 minutes (Hill et al., 2007). The role played by carnosine, or by $\beta$-alanine, is of great interest not only for untrained subjects: both compounds have a positive effect on strength in trained athletes, especially sprinters, rowers and body-builders as well (Baguet et al., 2010; Derave et al., 2010).

At physiological $\mathrm{pH}$, carnosine exerts a strong buffering action (stabilization of the degree of acidity) that is essential during muscle activity, usually associated with an acidification of the intracellular compartment (Hobson et al., 2012). Furthermore, carnosine has been recognized to have antioxidant prop- 
erties (Alhamdani et al., 2007), as well as stimulating actions on the immune system and various neurotransmitters (L-carnosine lowers neural activities of sympathetic nerves and enhances those of parasympathetic nerves) (Nagai et al., 2012). Some evidence indicates that carnosine is available and ready to exert its function inside the muscle fibres just after a few hours from its ingestion in high doses (Begum et al., 2005; Gardner et al., 1991).

Carnosine was recently shown to play a role in inflammation-reducing, pro-inflammatory cytokine release in vitro and in vivo. In particular, a study performed in murine microglial cells demonstrated that carnosine inhibits the synthesis of inflammatory mediators during lipopolysaccharide-induced inflammation (Fleisher-Berkovich et al., 2009). This protective effect of carnosine was also demonstrated in astroglial cells (Nicoletti et al., 2007). Moreover, carnosine prevents IL8 release, following $\mathrm{H}_{2} \mathrm{O}_{2}$ treatment, in human intestinal Caco-2 cells (Son et al., 2004). Lee et al. demonstrated that the intake of carnosine significantly suppresses the increase in inflammatory cytokines interleukin-6 (IL6) and tumour necrosis factor-alpha (TNF- $\alpha$ ) in diabetic mice (Lee et al., 2005).

Several studies have provided evidence that strenuous exercise induces increased expression of several pro- and anti-inflammatory cytokines in skeletal muscle and blood (Ostrowski et al., 1999). Among these, interleukin-6 seems to play a major role: IL6 expression is up-regulated during the exercise-induced inflammatory process and exerts an anti-inflammatory effect leading to the release of anti-inflammatory interleukins, such as interleukin-10 (IL10), and inhibiting TNF- $\alpha$ production (Capomaccio et al., 2011; Fischer, 2006; Petersen \& Pedersen, 2006). Large amounts of IL6 are produced in skeletal muscles and released into plasma in response to exercise and training, then muscle IL6 seems to stimulate the release of IL6 from peripheral blood mononuclear cells (PBMCs) (Fischer, 2006; Rhind et al., 2001).

In spite of the potential for a positive effect of acute ingestion of carnosine on high-intensity exercise performance, in a recent study (Invernizzi et al., 2013), we failed to demonstrate any improvement in performance during an intermittent high-intensity running test following acute ingestion (4 hours prior to the test) of $1 \mathrm{~g} \mathrm{~L}$-carnosine $+1 \mathrm{~g} \beta$-alanine, in comparison with that of the placebo group. These results seem to be in agreement with the data obtained by other authors who unsuccessfully tried to improve high-intensity cycling performance following acute ingestion of a soup with a high content in carnosine and anserine (Suzuki et al., 2006). It cannot be excluded that in these two previous studies the dose of carnosine administered to the participants was too low (13.5 and 6.2 $\mathrm{mg} \cdot \mathrm{Kg}^{-1}$ respectively).

The aim of this study was to evaluate whether acute ingestion of a high dosage of L-carnosine and $\beta$-alanine $(2 \mathrm{~g}+2 \mathrm{~g}$ respectively) would induce an improvement in performance during a running anaerobic sprint test (RAST) (Zagatto et al., 2009) in comparison with placebo. In addition, we investigated the effect of acute carnosine $+\beta$-alanine supplementation on the synthesis of exercise-induced cytokines, and in particular we studied the mRNA expression of interleukine- 6 and -10 in PBMCs in response to an anaerobic sprint.

On the basis of our previous results, in this study we decided to double the carnosine $+\beta$-alanine dosage (about $26.6 \mathrm{mg} \cdot \mathrm{Kg}^{-1}$ body weight each compound) and the physical effort (by asking the volunteers to repeat a second RAST following a recovery period). Our hypothesis was that this high dosage of carnosine $+\beta$-alanine (compared to the dosage of previous researches) would enhance performance, both in the first and especially in the second RAST, and affect the mRNA expression of interleukine- 6 and -10 in PBMCs.

The rationale of our hypothesis is based on the fact that a high acute (4 hours after the ingestion) dosage of carnosine and $\beta$-alanine $(2 \mathrm{~g}+2 \mathrm{~g})$ can increase the muscle content of carnosine such that the muscle buffering capacity raises. From an ethical point of view we decided to test the biological effects of our hypothesis first. Once this hypothesis is tested, a biopsy study would be reasonable and appropriate to look into the mechanisms responsible. Although this decision represents a limit for this study and can raise criticisms, we would like to avoid any invasive practice for our participants prior to having ascertained the enhancing effect on performance of an acute administration of carnosine $+\beta$-alanine.

\section{Materials and Methods}

\section{Subjects}

The participants were recruited in the Milan area in Italy, according to the following inclusion criteria: males aged between 20 and 30 years, no smokers, absence of chronic diseases or chronic drug treatment. The study was approved by the Ethical Committee of the University of Milan. All the subjects signed a written informed consent prior to participation, according to the Declaration of Helsinki. All the procedures used complied with the Good Clinical Practice (GCP) principles.

Twelve healthy male subjects were enrolled (age: $21 \pm 4$ years, height $181 \pm 3 \mathrm{~cm}$, body mass $75.1 \pm 4.6$, body fat percentage $12.0 \pm 3.6$, body mass index: $23.0 \pm 1.3 \mathrm{~kg} / \mathrm{m}^{2}$ ); all the participants were on a stable diet and were requested not to modify their diet during the period of the data collection. They were physically active and used to exercise on average $3.3 \pm$ 2.2 times a week for $1.5 \pm 1.0$ hours (carnosine $+\beta$-alanine group) or $2.2 \pm 1.2$ times a week for $2.5 \pm 1.3$ hours (placebo group) at vigorous and moderate intensities according to the International Physical Activity Questionnaire (IPAQ) (Craig et al., 2003). Most of the participants recruited for this study were soccer players accustomed to repeated intermittent all-out sprints. Prior to the beginning of the study, the volunteers participated in a familiarization session with the intermittent allout running sprint test used in this study.

\section{Study Design}

The study was conducted using a double blind, randomized, counterbalanced, cross-over, placebo-controlled design. The randomization and the preparation of treatment and placebo tablets were made by a person unrelated to the study. The treatment and placebo conditions were disclosed to the authors of the study only after the statistical analysis had been completed. All the participants underwent two double intermittent all-out running tests, separated by $30 \mathrm{~min}$ on two separate occasions: on the first test day, half of the participants (selected by a computer-assisted simple randomization procedure) ingested 2 $\mathrm{g}$ of L-carnosine $+2 \mathrm{~g}$ of $\beta$-alanine (DDM Carnosina, kindly provided by Omeopiacenza s.r.l., Pontenure-PC, Italy) 4 hours before the test, whereas the other half of the participants ingested placebo (made of microcrystalline cellulose, calcium phosphate, hydroxypropyl methylcellulose, magnesium stea- 
rate, silicon dioxideno and having the same appearance and taste as the tablets made of L-carnosine and $\beta$-alanine), and vice versa on the second test day ( 2 weeks later). The purity of the supplement was independently tested by the formulator of the tested product (Velleja Research) with the aim to exclude the presence of performance-enhancing compounds such as anabolic agents or stimulants. None of the participants reported the presence of possible side effects following the high acute ingestion of L-carnosine $+\beta$-alanine neither during the testing period nor in the next hours or the day after (in particular no one experienced any form of paresthesia that is recognised to be a likely symptom when high dosage of carnosine are administered). The 30 min interval between RAST 1 and RAST 2 was chosen so as to participants could rate the session RPE also after RAST 1.

Two weeks seems to be a more than sufficient washout period when carnosine is acutely administered to human subjects (Suzuki et al., 2006). In this study the participants treated with DDM Carnosina, even if the product contains $\beta$-Alanine, are simply called "carnosine group" or "carnosine condition". DDM Carnosine (a commercially-available supplement), in agreement with the Italian law number 169/2004, was notified to the Minister of Health in 2010 (Registration number: 53436) and registered as food supplement with both its active ingredients ( $\beta$-alanine and L-carnosine) belonging to the positive list of ingredients admitted as nutraceuticals, and with all of its excipients being food grade. DDM Carnosine and the placebo tablets were both manufactured by Procemsa Farmaceutici (Nichelino, Turin, Italy). According to the main formulator of the tested product (DDM Carnosina), the combination of carnosine $+\beta$-alanine was thought with the aim to maximize the carnosine content within the muscle cell. Theoretically, subjects with low level of carnosinase might directly benefit of the carnosine as is while subjects with higher level of carnosinase might take advantage of the fraction of beta alanine already present in each tablet $(250 \mathrm{mg})$ plus the amount coming from the splitting up of carnosine ( $\beta$-alanine and L-istidine).

\section{The Running-Based Anaerobic Sprint Test (RAST)}

A standardized 10-min warm-up, consisting of 5 min running at a moderate pace followed by 5 accelerations over a distance of $40 \mathrm{~m}$, interspersed with 1-min passive recovery, was completed by each participant prior to the beginning of the RAST. After 5-minute passive recovery, the participants started the first RAST (RAST 1). A thirty-minute passive recovery was observed before the beginning of the second RAST (RAST 2).

The RAST was performed as follows: starting from a standing position, on a $400 \mathrm{~m}$ track, each participant sprinted all-out over a distance of $35 \mathrm{~m}$ for six times (forth and back), interspersed by 10 -second passive recovery. The performance times were electronically recorded using photocells gates located 0.5 meters ahead of the start line and at a height of 1.3 meters from the ground. The $35 \mathrm{~m}$ were measured as the distance between the two photocells gates. During all the sprints the athletes were verbally encouraged by the researchers, in order to obtain the maximum performance. The RAST has been shown to be a valid and reliable tool for assessing anaerobic power during running (Zagatto et al., 2009). The outcome measures were 1) time to complete each section of $35 \mathrm{~m}, 2$ ) total time (the sum of the six partial times over the $35 \mathrm{~m}$ sections), 3) ratings of perceived exertion (RPE) and muscle pain (PAIN) immediately after the completion of each test (RPE 1 and PAIN 1, RPE 2 and PAIN 2 after RAST 1 and RAST 2 respectively) using the validated CR 10 Borg scale (Borg, 1998). Thirty minutes after the end of RAST 2, RPE was assessed again. The RPE values measured at this time were used to assess the internal load through the session RPE-based method (Foster et al., 2001; Impellizzeri et al., 2004).

\section{Blood Sample Collection and RNA Extraction}

One hour before the beginning of RAST 1 and one hour after the completion of RAST 2, $200 \mu$ l of blood were drawn from the fleshy pad of the fingertip of each subject.

Blood was collected in a heparinized tube, and total RNA from PBMCs was isolated using the PureLink Total RNA Blood Purification kit (Invitrogen) according to the instructions provided by the manufacturer.

\section{Reverse Transcription and Real-Time PCR}

The iScript cDNA Synthesis kit (Bio-Rad Laboratories) was used to reverse-transcribe $150 \mathrm{ng}$ RNA, and gene expression of IL6 and IL10 was assayed by real-time PCR.

Real-time PCR was performed by the iCycler thermal cycler (Bio-Rad Laboratories) using cDNA corresponding to 10ng total RNA as a template. PCR mixture included $0.2 \mu \mathrm{M}$ primers, $50 \mathrm{mM} \mathrm{KCl}, 20 \mathrm{mM}$ Tris/HCl pH 8.4, $0.8 \mathrm{mM}$ dNTPs, 0.7U iTaq DNA Polymerase, $3 \mathrm{mM} \mathrm{MgCl}_{2}$, and SYBR Green (iQ SYBR Green Supermix from Bio-Rad Laboratories) in a final volume of $20 \mu \mathrm{l}$. Amplification and real-time data acquisition were performed using the following cycle conditions: initial denaturation at $95^{\circ} \mathrm{C}$ for 3 minutes, followed by 40 cycles of 10 seconds each at $95^{\circ} \mathrm{C}$ and 30 seconds at $58^{\circ} \mathrm{C}$. The fold change in expression of the different genes was normalized to the expression of $\beta$-actin mRNA and was calculated by the equation $2^{-\Delta \Delta \mathrm{Ct}}$. All reactions were performed in triplicate. The primers used are reported in Table 1. The accuracy was monitored by the analysis of the melting curves.

\section{Statistical Analysis}

The normality of data distribution was preliminary checked by the Shapiro-Wilk's test. Differences in performance (total time given by the sum of each $35 \mathrm{~m}$ run), perceived exertion (RPE) and pain at the end of each RAST, between the two con-

Table 1.

Primers used for real-time PCR.

\begin{tabular}{crc}
\hline Primer & Forward & Reverse \\
\hline IL6 & 5'-CTTCGGTCCAGTTGCCTTC-3' & 5'-TGAAGAGGTGAGTGGCTGTCT-3' \\
IL10 & 5'-GCTGAGAACCAAGACCCAGA-3' & 5'-GCATTCTTCACCTGCTCCAC-3' \\
3-actin & 5'-CGACAGGATGCAGAAGGAG-3' & 5'-ACATCTGCTGGAAGGTGGA-3' \\
\hline
\end{tabular}


ditions (carnosine and placebo, main factor Condition) and the two RAST (RAST 1 and RAST 2, main factor Time), were tested using a $2 \times 2$ (Condition $\times$ Time) fully repeated multivariate ANOVA measures. Session RPE (30 minutes after the completion of RAST 2) between treatment and placebo was tested using a $2 \times 2$ (Condition $\times$ Time) fully repeated multivariate ANOVA.

A series of $2 \times 6$ (Condition $\times$ Time) fully repeated multivariate ANOVA measures were used to test the differences between the two conditions (treatment with carnosine or placebo, main factor condition) and the times to complete each bout of the RAST.

Changes in the relative expression of IL6 and IL10 between groups were calculated using a $2 \times 2$ (Condition $\times$ Time) fully repeated multivariate ANOVA measures, where the main factor Condition is represented by the carnosine or placebo administrations and the main factor Time is represented by the baseline (one hour prior to RAST 1) and post exercise (one hour after the completion of RAST 2) blood samples. Data are presented as the means \pm standard deviations (S.D.). The level of significance was set at $\alpha<0.05$.

\section{Results}

\section{Performance, Perceived Exertion and Pain}

No significant interactions were found for performance $(p=$ $0.472)$, RPE $(p=0.653$; carnosine $+\beta$-alanine: RAST $16.3 \pm$ 2.0 vs RAST $26.8 \pm 1.9$; placebo: RAST $1,6.3 \pm 2.2$ vs RAST $2,6.9 \pm 1.5)$ or pain $(p=0.099)$. However, the significant main effect of time on performance $(p<0.001)$ and pain $(p<0.05$; carnosine + $\beta$-alanine: RAST $1,3.0 \pm 2.1$ vs RAST $2,4.2 \pm 1.9$; placebo: RAST $1,3.0 \pm 1.8$ vs RAST $2,3.4 \pm 1.2$ ), showed that between RAST 1 and RAST 2 there was a decrease in performance (that is, a higher total time to complete RAST 2 in comparison with RAST 1) (Figure 1) and an augmented perceived pain at the end of the RAST 2 in comparison with RAST 1. No significant interaction and main effect of condition were detected for session RPE; on the contrary, the significant main effect of time $(p<0.01)$ showed an augmented session RPE over time (carnosine condition, $68 \pm 28$ a.u. vs $239 \pm 49$ a.u., post RAST 1 vs RAST 2 respectively; placebo, $62 \pm 17$ a.u. vs $233 \pm 52$ a.u. post RAST 1 vs post RAST 2 respectively).

During RAST 1 the time to complete each $35 \mathrm{~m}$ bout significantly increased in both conditions (main factor time, $p<$ 0.001 ) but without significant interaction (Condition $\times$ Time, $p$ $=0.281)($ Figure 2$)$. Both the main effects of time $(p<0.001)$ and condition $(p<0.05)$ of RAST 2 were found significant (Figure 3). The time to complete each $35 \mathrm{~m}$ bout progressively increased in both conditions; at group level, the significant main effect of condition $(p<0.05)$ stands for an overall faster time to complete the RAST 2 in the carnosine condition.

\section{IL6 and IL10 Gene Expression}

As shown in Figure 4 we found significant differences in the expression of IL 6 and IL10 before the beginning of RAST 1 and one hour after the completion of RAST 2. In particular, IL6 expression showed approximately a two-fold increase after

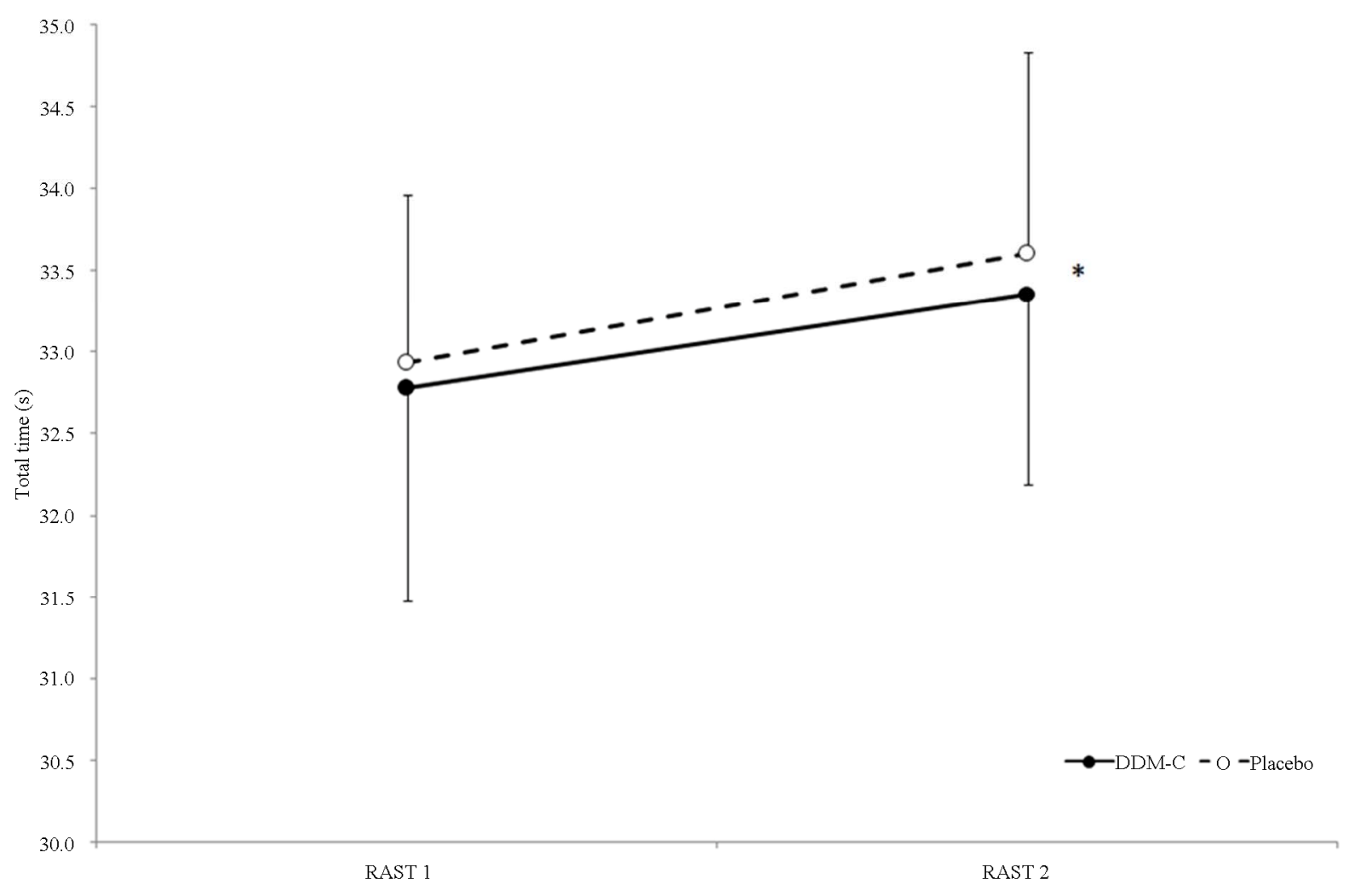

Figure 1.

Effects of DDM-C, compared to Placebo, on performance, expressed as total time to complete the Running Anaerobic Sprint Test (RAST), between RAST 1 and RAST 2; "Significant main effect of Time, $p<0.05$. DDM-C, L-carnosine $+\beta$-alanine. 


\section{P. L. INVERNIZZI ET AL.}

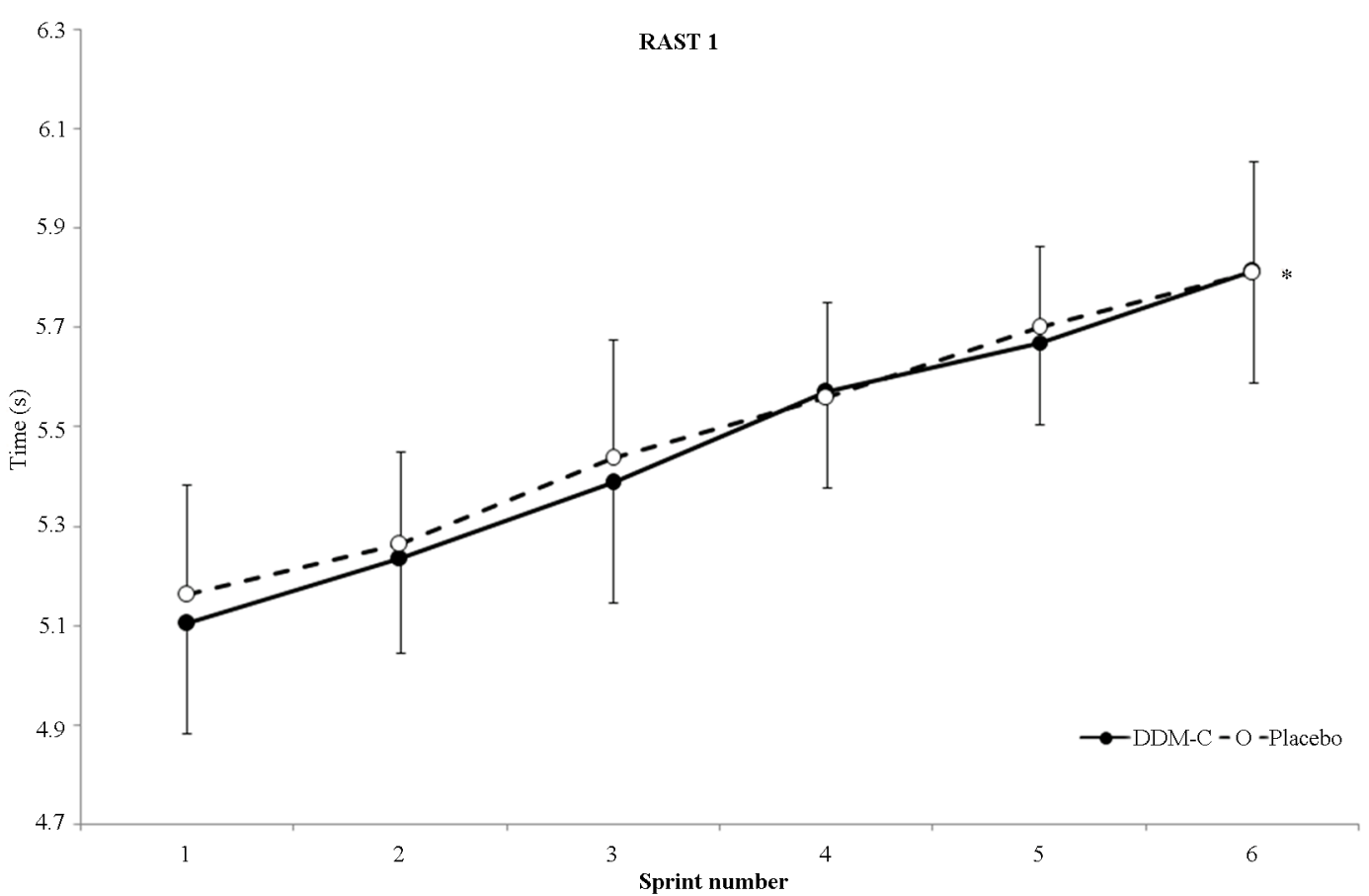

Figure 2.

Effects of DDM-C, compared to Placebo, on the time to complete each single sprint of RAST 1 (Running Anaerobic Sprint Test); ${ }^{*}$ Significant main effect of Time, $p<0.05$. DDM-C, L-carnosine $+\beta$-alanine.

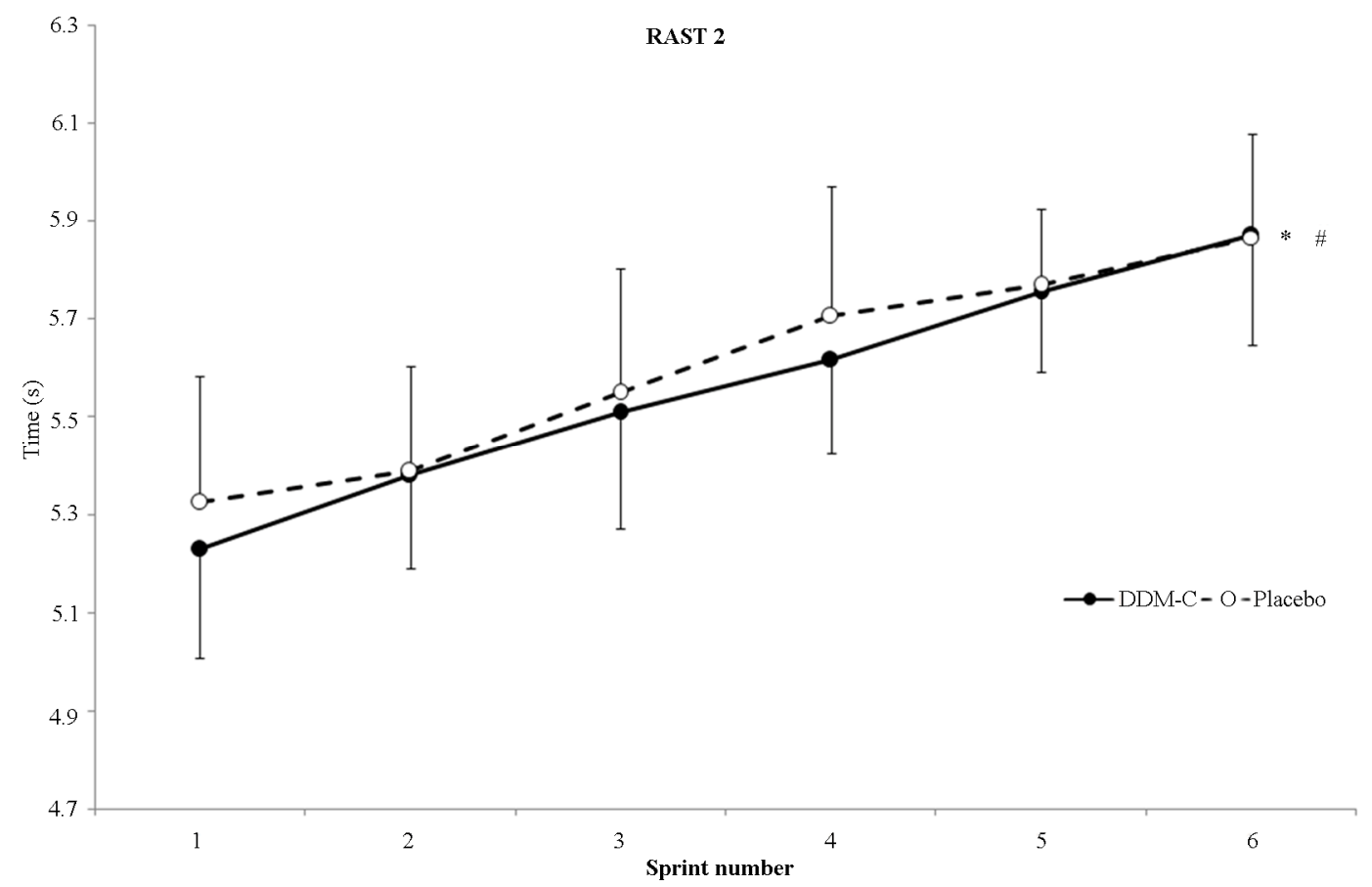

Figure 3.

Effects of DDM-C, compared to Placebo, on the time to complete each single sprint of RAST 2 (Running Anaerobic Sprint Test); "Significant main effect of Time, $p<0.05$; "Significant main effect of Condition, $p<0.05$. DDM-C, L-carnosine $+\beta$-alanine.

exercise (Figure 4(a)), whereas IL10 expression was reduced by approximately fifty per cent (Figure 4(b)). However, there were no statistically significant differences in IL6 and IL10 gene expression between the carnosine and placebo groups $(p>$
$0.05)$

\section{Discussion}

The purpose of this study was to determine if an acute ingestion of L-carnosine and $\beta$-alanine would improve performance 


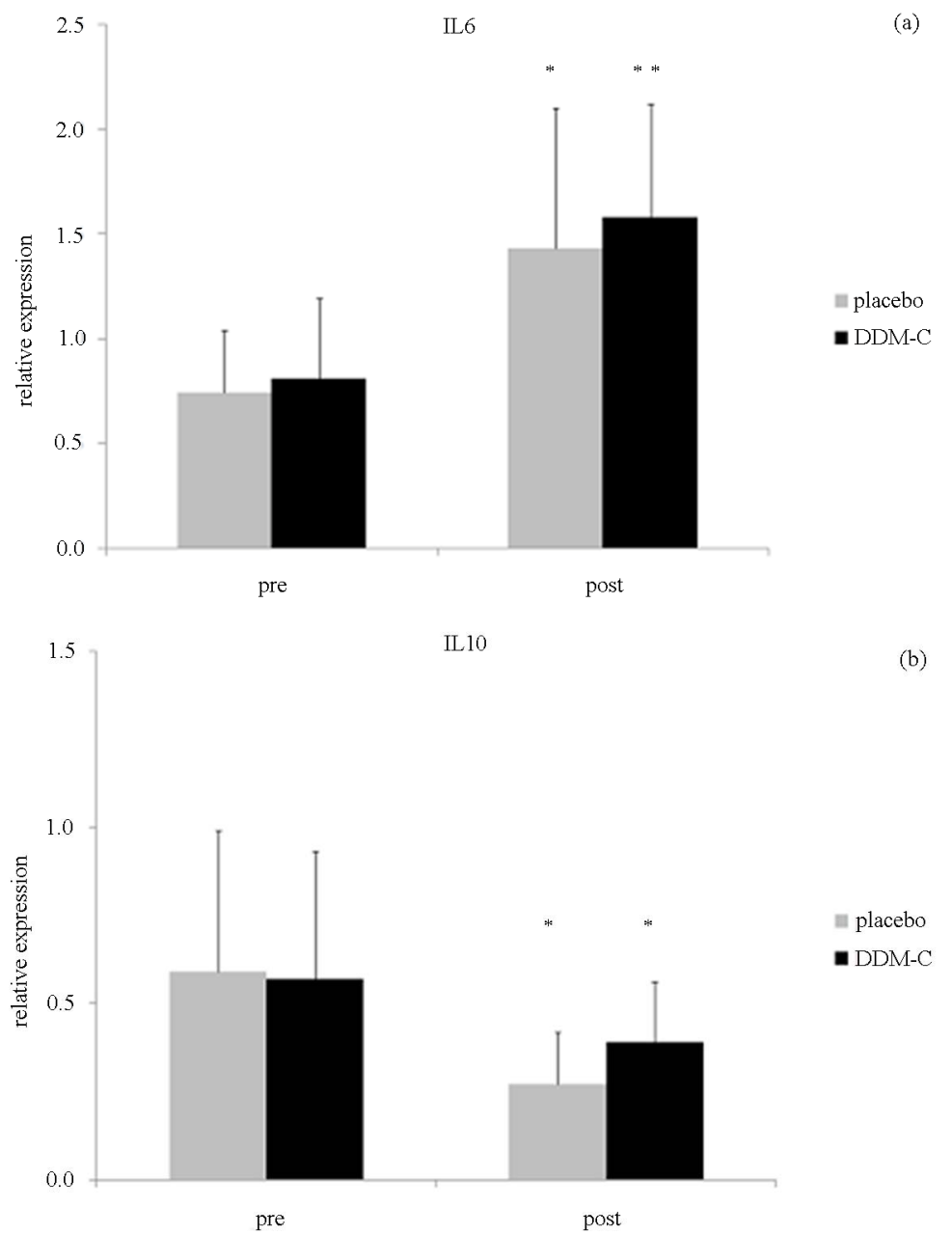

Figure 4.

Interleukin-6 (a) and -10 (b) mRNA expression by real time PCR before the beginning of RAST1 (pre) and one hour after completion of RAST 2 (post). Values are the means \pm SD. Significant main effect of Time ${ }^{*} p<0.05$ and ${ }^{* *} p<0.01$.

during an anaerobic sprint test. Moreover, we analysed whether this supplementation would affect exercise-induced inflammatory response in blood, in particular decreasing IL6 gene expression, therefore exerting a positive effect on performance.

The main findings of this study are that ingestion of a dose of carnosine $+\beta$-alanine $(2+2 \mathrm{~g})$, compared to a placebo condition, 4 hours prior to the exercise did not affect the overall performance or the first bout of an intermittent all-out running exercise but had an enhancing, albeit very low, effect on the second bout of the same intermittent exercise performed 30 minutes later. In addition, the large dose of carnosine ingested by the participants did not affect the IL6 or IL10 response to exercise in comparison with the placebo condition.

During RAST 1 , the time to complete each $35 \mathrm{~m}$ bout increased similarly in both conditions, with no difference in total time. During RAST 2, the trend was similar to RAST 1 but a likely small difference was detected between the two conditions.

Contrary to our hypothesis, performance was not improved following the high dosage of carnosine during RAST 1. Interestingly, during RAST 2 there was a very small enhancement in performance in the carnosine condition in comparison with placebo. It is likely that this effect is very modest and this issue is supported by the low-to-moderate partial eta squared value (partial $\eta^{2}=0.363$ ). However, from a practical point of view, just few hundredths of a second can represent a sufficient gap to make the difference between the winner and the second place in sprinting races of different sports (such as 200 and $400 \mathrm{~m}$ in the track and field, 50 and $100 \mathrm{~m}$ in swimming, 500 and 1000 $\mathrm{m}$ time trial in track cycling, etc.). We believe this is a concern not to be underestimated.

According to Suzuki et al. (2006) the possible enhancement in performance during RAST 2 might be due to the role played by the non-bicarbonate buffering system. Carnosine, within the muscle cells, can rapidly accept the protons produced during high-intensity exercise in favour of a preservation of the $\left[\mathrm{HCO}^{3-}\right]$. The lack of improvement in high-intensity cycling performance (Suzuki et al., 2006) was attributed to the limited buffering potential of the carnosine supplementation (dosage about $6.2 \mathrm{mg} \cdot \mathrm{Kg}^{-1}$ ). A higher dosage, adopted in our previous study (data not published), failed to improve performance. In this study a double dosage of carnosine failed again to show an improvement in performance during RAST 1 but a possible enhanced performance was highlighted in RAST 2. It may be 
supposed, therefore, that the carnosine buffering capacity can be exerted when the total duration of a high-intensity intermittent exercise is longer than $30-40$ seconds. Indeed, in the placebo condition any carnosine present in the muscle might have been depleted at the end of RAST 1 and was therefore lower during RAST 2. Such a circumstance did not likely occur in the carnosine condition. This hypothesis seems to be in harmony with the consideration proposed in a recent meta-analysis (Baguet et al., 2010) that shows how the effect of chronic supplementation of $\beta$-alanine is particularly effective in exercises lasting from 60 to $240 \mathrm{~s}$. It can be believed that similar mechanisms occur also following acute ingestion of L-carnosine + $\beta$-alanine: in the present study the total duration of exercise is around $60-65 \mathrm{~s}$ and the higher amount of L-carnosine and $\beta$-alanine administered acutely to the participants might have significantly increased the muscle content of carnosine and the muscle buffering capacity.

A further and alternative mechanism through which muscle carnosine can improve muscle performance (Dutka \& Lamb, 2004 ) is the increased $\mathrm{Ca}^{2+}$ sensitivity exerted on the contractile fibres. A high dosage of carnosine, like that used in this study, might have had an effect on the $\mathrm{Ca}^{2+}$ sensitivity and was likely to have contributed to lowering times in some of the sprints during RAST 2.

In addition, due to the potential antioxidant activity of carnosine (Kohen et al., 1988; Decker et al., 2000), some not yet well understood mechanisms might have contributed to positively affect short term all out performance in the carnosine group of the present study.

As far as performance is concerned, this study is in agreement with a study on cycling (Suzuki et al., 2002), in which carnosine was supposed to have a positive effect towards the end of a Wingate test. However, this study is in contrast with previous results showing a lack of increase in intermittent cycling performance after the ingestion of carnosine, although it was effective in the preservation of blood $\left[\mathrm{HCO}^{-}\right]$(Suzuki et al., 2006). On the whole, it is necessary to point to some differences among these studies to understand the diverse and similar results. First of all, the type of exercise: in this study we used running while in the other studies it was cycling (Suzuki et al., 2002; Suzuki et al., 2006). In addition, the test was based on continuous versus intermittent cycling. Second, the amount of carnosine administered to the subjects was higher than that used in the two studies on cycling (Suzuki et al., 2002; Suzuki et al., 2006) and in our previous study (data not published).

Although we observed a significant difference in interleukin gene expression prior to, and following, exercise, we did not find a correlation with L-carnosine- $\beta$-alanine and placebo treatments. The marked exercise-associated increase in IL6 gene expression in PBMCs is in agreement with other studies (Rhind et al., 2001; Sander et al., 1998; Hagiwara et al., 1995), but supplementation failed to modify interleukin expression and in particular the IL6/IL10 ratio. It is conceivable that an acute supplementation may not be enough to modulate PBMC cytokine production or to modify IL6 and IL10 expression, and probably a prolonged period of supplementation could be needed to have a significant anti-inflammatory effect.

One of the main limits of our study is the lack of blood analysis, which could have been evidence of potential differences in lactate, $\mathrm{pH}$ or $\left[\mathrm{HCO}^{-}\right]$and, consequently, provide a better explanation of the mechanisms underpinning the possible positive effect of carnosine on performance.

\section{Conclusion}

In conclusion, the ingestion of L-carnosine and $\beta$-alanine (2 $\mathrm{g}+2 \mathrm{~g}) 4$ hours prior to an intermittent all-out anaerobic exercise does not have any effect on the PBMCs interleukin- 6 and -10 gene expression and on a first trial of intermittent running performance but increases overall performance during a second trial run $30 \mathrm{~min}$ after the first one. The mechanisms of this increase in performance could be ascribed to a long term enhanced muscle buffering capacity exerted by the acute ingestion of L-carnosine and $\beta$-alanine.

\section{Acknowledgements}

The Authors wish to thanks all the participants for their enthusiasm and commitment during data collection. A particular thank to Andrea Bosio for his valuable feedback during the design of the study and the preparation of this manuscript.

The authors, but Di Pierro F. who is the main formulator of the tested product (DDM Carnosina), declare no conflicts of interest that are directly relevant to the content of this article.

\section{REFERENCES}

Alhamdani, M. S., Al-Azzawie, H. F., \& Abbas, F. K. (2007). Decreased formation of advanced glycation end-products in peritoneal fluid by carnosine and related peptides. Peritoneal Dialysis International, 27, 86-89.

Baguet, A., Bourgois, J., Vanhee, L., Achten, E., \& Derave, W. (2010). Important role of muscle carnosine in rowing performance. Journal of Applied Physiology, 109, 1096-1101. http://dx.doi.org/10.1152/japplphysiol.00141.2010

Begum, G., Cunliffe, A., \& Leveritt, M. (2005). Physiological role of carnosine in contracting muscle. International Journal of Sport $\mathrm{Nu}$ trition and Exercise Metabolism, 15, 493-514.

Borg, G. (1998). Borg's perceived exertion and pain scales. Champaign, IL: Human Kinetics.

Capomaccio, S., Cappelli, K., Spinsanti, G., Mencarelli, M., Muscettola, M., Felicetti, M., Verini Supplizi A., \& Bonifazi, M. (2011). Athletic humans and horses: Comparative analysis of interleukin-6 (IL-6) and IL-6 receptor (IL-6R) expression in peripheral blood mononuclear cells in trained and untrained subjects at rest. BMC Physiology, 11, 3. http://dx.doi.org/10.1186/1472-6793-11-3

Craig, C. L., Marshall, A. L., Sjostrom, M., Bauman, A. E., Booth, M. L., Ainsworth, B. E., Pratt, M., Ekelund, U., Yngve, A., Sallis, J. F., \& Oja, P. (2003). International physical activity questionnaire: 12country reliability and validity. Medicine \& Science in Sports \& Exercise, 35, 1381-1395.

http://dx.doi.org/10.1249/01.MSS.0000078924.61453.FB

Decker, E. A., Livisay, S. A., \& Zhou, S. (2000). Re-evaluation of the antioxidant activity of purified carnosine. Biochemistry (Moscow), 65, 766-770.

Derave, W., Ozdemir, M. S., Harris, R. C., Pottier, A., Reyngoudt, H., Koppo, K., et al. (2007). beta-Alanine supplementation augments muscle carnosine content and attenuates fatigue during repeated isokinetic contraction bouts in trained sprinters. Journal of Applied Physiology, 103, 1736-1743. http://dx.doi.org/10.1152/japplphysiol.00397.2007

Derave, W., Everaert, I., Beeckman, S., \& Baguet, A. (2010). Muscle carnosine metabolism and beta-alanine supplementation in relation to exercise and training. Sports Medicine, 40, 247-263. http://dx.doi.org/10.2165/11530310-000000000-00000

Dutka, T. L., \& Lamb, G. D. (2004). Effect of carnosine on excitationcontraction coupling in mechanically-skinned rat skeletal muscle. Journal of Muscle Research and Cell Motility, 25, 203-213. http://dx.doi.org/10.1023/B:JURE.0000038265.37022.c5

Fischer, C. P. (2006). Interleukin-6 in acute exercise and training: What is the biological relevance? Exercise Immunology Review, 12, 6-33. 


\section{P. L. INVERNIZZI ET AL}

Fleisher-Berkovich, S., Abramovitch-Dahan, C., Ben-Shabat, S., Apte, R., \& Beit-Yannai, E. (2009). Inhibitory effect of carnosine and Nacetyl carnosine on LPS-induced microglial oxidative stress and inflammation. Peptides, 30, 1306-1312. http://dx.doi.org/10.1016/j.peptides.2009.04.003

Foster, C., Florhaug, J. A., Franklin, J., Gottschall, L., Hrovatin, L. A., Parker, S., Doleshal, P., \& Dodge, C. (2001). A new approach to monitoring exercise training. Journal of Strength and Conditioning Research, 15, 109-115.

Gardner, M. L., Illingworth, K. M., et al. (1991). Intestinal absorption of the intact peptide carnosine in man, and comparison with intestinal permeability to lactulose. Journal of Physiology, 439, 411-22.

Hagiwara, E., Abbasi, F., Mor, G., Ishigatsubo, Y., \& Klinman, D. M. (1995). Phenotype and frequency of cells secreting IL-2, IL-4, IL-6, IL-10, IFN and TNF- $\alpha$ in human peripheral blood. Cytokine, 7, 815822. http://dx.doi.org/10.1006/cyto.1995.0098

Harris, R. C., Wise, J. A., Price, K. A., Kim, H. J., Kim, C. K., \& Sale, C. (2012). Determinants of muscle carnosine content. Amino Acids, 43, 5-12. http://dx.doi.org/10.1007/s00726-012-1233-y

Hill, C. A., Harris, R. C., Kim, H. J., Harris, B. D., Sale, C., Boobis, L. H., et al. (2007). Influence of beta-alanine supplementation on skeletal muscle carnosine concentrations and high intensity cycling capacity. Amino Acids, 32, 225-233.

http://dx.doi.org/10.1007/s00726-006-0364-4

Hobson, R. M., Saunders, B., Ball, G., Harris, R. C., \& Sale, C. (2012). Effects of beta-alanine supplementation on exercise performance: A meta-analysis. Amino Acids, 43, 25-37.

http://dx.doi.org/10.1007/s00726-011-1200-Z

Impellizzeri, F. M., Rampinini, E., Coutts, A. J., Sassi, A., \& Marcora, S. M. (2004). Use of RPE-based training load in soccer. Medicine \& Science in Sports \& Exercise, 36, 1042-1047. http://dx.doi.org/10.1249/01.MSS.0000128199.23901.2F

Invernizzi, P.L.,Benedini, S., Saronni, S., Merati, G., \& Bosio, A. (2013). The acute administration of Carnosine and beta-Alanine does not improve running anaerobic performance and has no effect on the metabolic response to exercise. Advances in Physical Education, 3, 169-174. http://dx.doi.org/10.4236/ape.2013.34028

Jagim, A. R., Wright, G. A., Brice, A. G., \& Doberstein, S. T. (2013). Effects of Beta-alanine supplementation on sprint endurance. Journal of Strength and Conditioning Research, 27, 526-532. http://dx.doi.org/10.1519/JSC.0b013e318256bedc

Kohen, R., Yamamoto, Y., Cundy, K. C., \& Ames, B. N. (1988). Antioxidant activity of carnosine, homocarnosine, and anserine present in muscle and brain Proceedings of the National Academy of Sciences USA, 85, 3175-3179.

Lee, Y. T., Hsu, C. C., Lin, M. H., Liu, K. S., \& Yin, M. C. (2005). Histidine and carnosine delay diabetic deterioration in mice and protect human low density lipoprotein against oxidation and glycation. European Journal of Pharmacology, 513, 145-150. http://dx.doi.org/10.1016/j.ejphar.2005.02.010

Margolis, F. L., Grillo, M., Kawano, T., \& Farbman, A. I. (1985). Carnosine synthesis in olfactory tissue during ontogeny: Effect of exogenous beta-alanine. Journal of Neurochemistry, 44, 1459-1464. http://dx.doi.org/10.1111/j.1471-4159.1985.tb08783.x

Nagai, K., Tanida, M., Niijima, A., Tsuruoka, N., Kiso, Y., Horii, et al. (2012). Role of L-carnosine in the control of blood glucose, blood pressure, thermogenesis, and lipolysis by autonomic nerves in rats: Involvement of the circadian clock and histamine. Amino Acids, 43, 97-109. http://dx.doi.org/10.1007/s00726-012-1251-9

Nicoletti, V. G., Santoro, A. M., Grasso, G., Vagliasindi, L. I., Giuffrida, M. L., Cuppari, et al. (2007). Carnosine interaction with nitric oxide and astroglial cell protection. Journal of Neuroscience Research, 85, 2239-2245. http://dx.doi.org/10.1002/jnr.21365

Ostrowski, K., Rohde, T., Asp, S., Schjerling, P., \& Pedersen, B. K. (1999). Pro- and anti-inflammatory cytokine balance in strenuous exercise in humans. Journal of Physiology, 515, 287-291. http://dx.doi.org/10.1111/j.1469-7793.1999.287ad.x

Petersen, A. M., \& Pedersen, B. K. (2006). The role of IL-6 in mediating the anti-inflammatory effects of exercise. Journal of Physiology and Pharmacologyl, 57, 43-51.

Rhind, S. G., Castellani, J. W., Brenner, I. K., Shephard, R. J., Zamecnik, J., Montain, S. J., et al. (2001). Intracellular monocyte and serum cytokine expression is modulated by exhausting exercise and cold exposure. American Journal of Physiology-Regulatory, Integrative and Comparative Physiology, 281, R66-75.

Sander, B., Andersson, J. \& Andersson, U. (1991) Assessment of cytokines by immunofluorescence and the paraformaldehyde-saponin procedure. Immunological Reviews, 119, 65-93. http://dx.doi.org/10.1111/j.1600-065X.1991.tb00578.x

Son, D. O., Satsu, H., Kiso, Y., \& Shimizu, M. (2004). Characterization of carnosine uptake and its physiological function in human intestinal epithelial Caco-2 cells. Biofactors, 21, 395-398. http://dx.doi.org/10.1002/biof.552210177

Suzuki, Y., Ito, O., Mukai, N., Takahashi, H., \& Takamatsu, K. (2002). High level of skeletal muscle carnosine contributes to the latter half of exercise performance during 30-s maximal cycle ergometer sprinting. Japanese Journal of Physiology, 52, 199-205. http://dx.doi.org/10.2170/jiphysiol.52.199

Suzuki, Y., Nakao, T., Maemura, H., Sato, M., Kamahara, K., Morimatsu, F., \& Takamatsu, K. (2006). Carnosine and anserine ingestion enhances contribution of nonbicarbonate buffering. Medicine \& Science in Sports \& Exercise, 38, 334-338.

Zagatto, A. M., Beck, W. R., \& Gobatto, C. A. (2009). Validity of the running anaerobic sprint test for assessing anaerobic power and predicting short-distance performances. Journal of Strength \& Conditioning Research, 23, 1820-1827.

http://dx.doi.org/10.1519/JSC.0b013e3181b3df32 